Schweiz. Z. Path. Bakt. 1944;7:I-IV

\title{
Contents, Vol. 7, 1944
}

Schweizerische Zeitschrift

für

Pathologie und Bakteriologie

Revue Suisse de Pathologie et de Bactériologie

Unter Mitarbeit von:

C. Hallauer P. Hauduroy H. v. Meyenburg J.L.Nicod Th. Reh

Bern Lausanne ZurichLausanne Geneve

E. Rutishauser J. Tomcsik C. Wegelin A. Werthemann

Geneve Basel Bern Basel

Herausgegeben von:

A. v. Albertini - A. Grumbach - H. Mooser Zurich

Vol. VII

s.lא

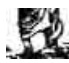

*."*o

1944

BASEL (Schweiz) S. KARGER NEWYORK

AUe Rechte vorbehalten

Printed in Switzerland.

Cliches: Aberegg-Steiner \& Cie. AC, Bern.

Druck von Friedrich Reinhardt in Basel.

Index

Armangué, M., Quelques données techniques sur Faction de bactéricides

su $\gamma$ le mycobactérium tuberculosis dans les crachat $\$ / 8$. . . 351

Quelques observations sur la toxinogénèse du Corynebacterium diphtheriae vide Reh, Th.

Armangué, M., et Dedie, 0., Sur la conservation des souches d'Eber-

thella typhosa type «VI» 373

Armangué, M., Novel, E., et Dedie, 0., Recherches immunologiques au

sujet de l'édestine, de la clupéine et de la salmine 505

Aufdermau, M., und Grumbach, A., Serologische Keuchhustendiagno-

stik $\quad 520$

Baer, J. G., Immunité et reactions immunitaires chez les Invertébrés . 442 Berblinger, W., Zur

Kenntnis der Pinealocytome nebst Bemerkungen

über die cerebrogene Frühreife $\quad 107$

Bernoulli, P., Untersuchungeii über den Wirkungsmechanismus des

Emetins bei bakteriellen Infektionen. I. Versuche in vitro . . . 525

- $\quad$ vide Bloch, $\mathrm{H}$. 
Biro, L., Die Beeinflußbarkeit von Schutzimpfungen durch Ergone . 624 BlaSkovic, D., Ueber die Wirkung einiger Sulfanilamide au $\cdot f$ expe'rimen-

telle Infektionen mit dem b-Typus des H. influenzae Pfeiffer . 614 Bloch, H., Ueber den

Stoffwechsel von Tuberkelbazilfen. 2. Mitteilung:

Die Ersetzbarkeit von Glycerin $\quad 589$

Bloch, H., und Bernoulli, P., Fermentchemische Untersuchungen an

sulfonamidresistenten Pneumokokken 394

Bouvier, G., vide Hauduroy, P.

Brunner, Th., vide Frei, W.

Brütsch, H., Das arterielle Gefäßinjektionsbild gesunder und kranker

Nieren in verschiedenen Altersstufen 560

Büttler, $\mid \mathrm{V}$, Die Bedeutung der Spitzennarbenblasen für die Entstehung

des idiopathischen Spontanpneumothorax 251

Dedie, O., vide Armangué, M.

- $\quad$ vide Reh, Th.

Egger, P., Endocarditis obliterans. Ihre Beziehungen zur Endocarditis

parietalis fibroplastica mit Bmteosinophilie und zum Morbus Buer-

ger 237

Eha, M., Wabenlunge. Beitrag zur Pathogenese cystischer Lungenverän-

derungen 20

Eöllös, Z., vide Ivánovics, G.

Faragó, P., Untersuchungen über die serologischen Eigenschaften der

spezifischen Substanz des Keuchhustenbazillus 603

Frei, W., und Brunner, Th., Beziehungen zwsichen Zellteilung und Oxy-

dation bei Bakterien 388

Fust, B., vide Hallauer, C.

Gäumann, E., Inimunreaktdonen und Immunität bei Pflanzen . . 407

Grumbach, A., Ueber das Wesen der Sul-fonamidempfindlichkeit hoch-

virulenФer Streptokokken 402

- vide Aufdermaur, M.

Hallauer, C, Immunität und Immunitätsreaktionen bei Säugetieren . . 463

Hallauer, C, und Fust, B., Ueber den Einfl · uß von Aspergillin und Peni

cillin auf Virusarten 380

Hauduroy, P., Bouvier, G., et Rosset, W., Nouvelle technique de décou-

verte des bacilles tuberculeux dans lies produits pathologiques . 346

Janiak, M., vide Zollikofer, E.

Jeney, A. v., und Korpássy, B. v., Ueber eine tumorartige Spontanerkran-

kung der pericoecalen Lymphknoten bei weißen Ratten ... 55

Ipsen, J., Quantitative Studien über mäusepathogene Fleckfieberrickett-

sien 129

Ivánovics, G., und Eöllös, Z., Die Bedeutung der PantothensäUГe, jjej

Ruhrbazillen 73

Karrer, P., Kurze Ausschnitte aus der Chemíe und Biochemie der Vit.

amine: Nicotinsäureamid, B \pm , B2, B $\beta$, Pantothensäure und Biotin 303

Kolonits, B., und Orbán, St., Behandlung der experimentellen Dysenterie

mit embryonalem Leberextrakt 
- $\quad$ Untersuchungen üb€ $\Gamma$ die antiseptiscbe Wirkung der Schieferölprä-

parate 152

Korpássy, B. v., vide Jeney, A. v.

Ladewig, P., und Serf, ü., Uebe $\Gamma$ brancbiogene Hemmungsmißbildungen 1

Moeschlin, S., und Rohr, K., Aplastische Anaemic mit jahrelangem vol·1-

ständigem Fehlen der Erythroblasten (Erythroblastophthise) . . 274 Mooser, H., Die Infektion der weißen Maus mit dem Erreger dee klas-

sischen Fleckfiebers 358

Novel, E., vide Armangué, M.

vide Reh, Th.

vide Kolonits, B.

Orbán, S., Polyvalenter antidysenФerischer Schutzimpfstoff .... 535 Pletscher, A., Ueber

imtrahepatische Varizen der Vena portae mit pro-

gred $>$ ienfer Pfortadê̂"thrombose (Hierzu Tafel I) 93

Pulver, R., und Sufer, R., Untersuchungen über den Wirkungsmechanis-

mus von Nt-3,4-Dimethylbenzoyl-sulfanilamid 398

Regamey, R., La dissociation de C. diphtheriae Park-Williams No 8.366

- $\quad$ Etude experimental ${ }^{\wedge}$ sur la résorption et $\Gamma$ élimination du serum

antiiétanique chez $1 \cdot e$ lapin ... $\quad 500$

Reh, Th., Armangué, M., Novel, E., et Dedie, O., Quelques recherches

sur les faits rapportés par 1·e Dr Seemüller. «Ueber ein paradoxes

serologisches Phänomen: Giftiges Serum wird durch Zusatz von

Gift ungiftig» 481

Reubi, F., Les vaisseaux et les gl-andes endocrines dans la neurofibromatose. Le syndrome sympathicotomique dans la maladie de Reck-

linghausen 168

Ritter, XV., Beitrag zur Kenmtnis der Laktoflavinbildung durch Eremo-

thecium Ashbyii Guill370

Rochat, R., Constatations sur la presence de corpuscules de Hassal intra-

kystiques 13

Rohr, K., vide Moeschlin, S.

Ro.iset, W., vide Hauduroy, P.

Schmid, G., Beitrag zur aktiven Immunisierung gegen die Flechten der

Pferde ... 496

Schopfer, W. H., Les vitamines, facteurs de croissance pour ks micro-

organismes 314

Seemüller, H., Experimentell·e Beiträge zur Aufdeckung des Diphtherie-

Immunitätsmechanismus $1 \quad 468$

Sert, D., vide Ladewig, P.

Skorpil, F., Zur Frage der Entstehung von Lamprocyten in der Brust-

drüse 552

Suter, R., vide Pulver, R.

Wagner, V., Zur Kenntnis der Pathogenität des Streptococcus lactis . 163

Wunderly, Ch., Ueber die Kinelik der Haemolyse durch Lecithin . . 285

Zínk, A., Studien über die Maul- und Klauenseuche in Brasilien.

I. Pathogenität einiger in Brasi $\Gamma$ ien gesammelter Stämme und 
natürliche Immunität des einheimíschen Viehs ...

II. Virulenz und Häufigkeit des Typs «O» 41

III. Ergebnisse und Schwierigkeiten mti der Vakzine nach Schmidt-

Waldmann 46

Zollikofer, E., und Janiak, M., Zur serologischen Differenzierung des

Streptococcus thermophilus

65

GESELLSCHAFTSBERICHTE - SOCIETY TRANSACTIONS - SOCIÉTÉS

Verhandlungen der Schweizerischen Mikrobiologischen Gesell·schaft.

3. Jahresversammlung in Zurich, 24. und 25. Juni 1944. Rapports

de la Société suisse de Microbiologie. 3e assemblée annuelle à $\mathrm{Zu}$

rich les 24 et 25 juin $1944 \quad 301$

BUCHBESPRECHUNGEN - BOOKS REVIEW - LIVRES NOUVEAUX 63,128, 236 